\title{
Activity of SNP variants of human uracil-DNA glycosylases SMUG1 and MBD4
}

\author{
Irina V. Alekseeva \\ Institute of Chemical Biology and \\ Fundamental Medicine \\ Novosibirsk, Russia \\ Irina.Alekseeva@niboch.nsc.ru \\ Nikita A. Kuznetsov \\ Institute of Chemical Biology and \\ Fundamental Medicine \\ Novosibirsk, Russia \\ Nikita.Kuznetsov@niboch.nsc.ru
}

\author{
Artemiy S. Bakman \\ Institute of Chemical Biology and \\ Fundamental Medicine \\ Novosibirsk, Russia \\ art-bakman@yandex.ru
}

\author{
Olga S. Fedorova \\ Institute of Chemical Biology and \\ Fundamental Medicine \\ Novosibirsk, Russia \\ fedorova@niboch.nsc.ru
}

\begin{abstract}
Human uracil-DNA glycosylases SMUG1 and MBD4 are crucial enzymes of the base excision repair pathway, responsible for uracil repair. It has been hypothesized that single-nucleotide polymorphic variants of SMUG1 and MBD4 might be responsible for an increased risk of some types of cancer. In the present work, analysis of SNPs of these uracilDNA glycosylases was performed to select the set of variants having substitutions of amino acid residues on the surface of the enzyme globule and in the DNA-binding site, thereby affecting protein-protein interactions or the catalytic reaction, respectively. Conformational dynamics and catalytic activities of four SMUG1 and four MBD4 SNP variants were examined. The conformational changes in the molecules of enzymes and in a DNA substrate were recorded as fluorescence changes of Trp and 2-aminopurine residues as well as FRET-signal.
\end{abstract}

Keywords - DNA repair, human uracil-DNA glycosylase, stopped-flow enzyme kinetics, fluorescence

Base excision repair (BER) is a key genome maintenance pathway that removes endogenously damaged DNA bases that arise in cells at very high levels on a daily basis. Amino acid substitutions in BER enzymes associated with singlenucleotide polymorphisms (SNPs) are widespread in the human population. The relation between SNP variants of BER proteins and etiology of some human diseases requires further investigation. It is known that some polymorphisms of the human AP-endonuclease or 8-oxoguanine-DNA glycosylase genes lead to decrease in the enzymatic activity. Such variants of BER enzymes in the human population can be associated with an increased risk of diseases $[1,2]$. The association of SNPs of BER genes with some diseases was recently discussed in a number of reviews [3,4]. It should be noted that a decrease in the functional activity of individual BER enzymes and disruption of coordination between them or with protein factors such as XRCC1 (which acts as a scaffold protein in the BER pathway) can have severe negative consequences for the human body [5-7].

Uracil in DNA can be introduced via two mechanisms, deamination of cytosine and misincorporation of dUMP during replication. Deamination of cytosine has been calculated from measured deamination rates to occur at a rate of 100-500 per human cell/day to yield mutagenic U/G mispairs. Uracil may also appear as a consequence of misincorporation of dUMP instead of dTMP during replication, resulting in a U/A base pair. Human single-strand selective monofunctional uracil-DNA glycosylase SMUG1, thymine-DNA glycosylase (TDG), uracil-DNA glycosylase (UNG), and methyl-CpG-binding domain (MBD4) have high affinity to uracil-containing DNA and catalyze a cleavage of the N-glycosidic bond involving a target base. They also possess individual substrate specificity to a single-stranded
DNA substrate, $\mathrm{T} / \mathrm{G}$ or $\mathrm{U} / \mathrm{G}$ mismatches, and to some oxidized bases such as 5-hydroxymethyluracil, 5-formyluracil, and 5hydroxyuracil. Interestingly to note, UNG, TDG, and SMUG1 belong to different families (I, II, and III, respectively) of the large uracil-DNA glycosylase structural superfamily [8], whereas MBD4 belongs to structural superfamily $\mathrm{HhH}$ (helixhairpin-helix) [9] of DNA-binding proteins. Given that human has four uracil-DNA glycosylases, indicating importance of uracil repair, we have conducted a systematic analysis of single-nucleotide polymorphism (SNP) variants of two uracil-DNA glycosylases SMUG1 and MBD4. Our aim was to determine the influence of known SNPs on the conformation and activity of human SMUG1 and MBD4. Using the NCBI dbSNP database (http://www.ncbi.nlm.nih.gov/SNP/), exonic polymorphic variants of the human SMUG1 and MBD4 were selected. Subsequent analysis of potential importance of an SNP was based on a principle of a maximal change in the chemical nature of the side group of the amino acid encoded by a corresponding nucleotide triplet. Spatial location of the amino acid residue close to the DNA-binding site and/or active site of the enzyme was also taken into account. Four SMUG1 (G90C, P240H, N244S and N248Y) and four MBD4 (S470L, G507S, R512W and H557D) SNP variants were chosen for stopped-flow analyses of conformational dynamics and kinetics of interaction with DNA substrates. The activity of SNP variants was studied by direct PAGE analysis of the kinetics of accumulation of products. The conformational transitions in the molecules of WT enzymes and its SNP variants in the course of interaction with DNA-substrate were monitored as changes in the intrinsic Trp fluorescence. To determine the effect of amino acid substitutions on the conformational changes in the DNA substrate, the changes in fluorescence intensity of aPu residues in the model substrate were monitored as well. A FAM/BHQ1-labeled substrate was also subjected to FRET analysis of DNA distortion processes and of the cleavage reaction. Obtained results made it possible to determine the kinetic mechanism underlying the interactions of the SNP variants with DNA substrates, to calculate the rate constants of the elementary stages, and to identify the stages of the process affected by mutation.

\section{ACKNOWLEDGMENT}

This study was supported partially by the Russian Ministry of Science and Education (project \#AAAA-A17117020210022-4). A pre-steady-state kinetic analysis of the enzyme interaction with DNA substrates was supported by a grant of the Russian Science No. 16-14-10038. 


\section{REFERENCES}

[1] Karahalil, B.; Bohr, V. A.; Wilson, D. M. Impact of DNA polymorphisms in key DNA base excision repair proteins on cancer risk. Hum. Exp. Toxicol. 2012, doi:10.1177/0960327112444476.

[2] Sweasy, J. B.; Lang, T. M.; DiMaio, D. Is base excision repair a tumor suppressor mechanism? Cell Cycle 2006, 5, 250-259, doi:Doi 10.4161/Cc.5.3.2414

[3] Wallace, S. S.; Murphy, D. L.; Sweasy, J. B. Base excision repair and cancer. Cancer Lett. 2012.

[4] Marsden, C. G.; Dragon, J. A.; Wallace, S. S.; Sweasy, J. B. Base Excision Repair Variants in Cancer. Methods Enzym. 2017, 591, 119 157, doi:10.1016/bs.mie.2017.03.003.

[5] Abbotts, R.; Wilson, D. M. Coordination of DNA single strand break repair. Free Radic. Biol. Med. 2017, 107, 228-244, doi:10.1016/j.freeradbiomed.2016.11.039.

[6] Kwiatkowski, D.; Czarny, P.; Galecki, P.; Bachurska, A.; Talarowska, M.; Orzechowska, A.; Bobinska, K.; Bielecka-Kowalska, A.; Pietras, T.; Szemraj, J.; Maes, M.; Sliwinski, T. Variants of Base Excision Repair Genes MUTYH , PARP1 and XRCC1 in Alzheimer's Disease
Risk.

Neuropsychobiology

2015 ,

71 ,

$176-186$, doi:10.1159/000381985.

[7] Czarny, P.; Kwiatkowski, D.; Toma, M.; Kubiak, J.; Sliwinska, A.; Talarowska, M.; Szemraj, J.; Maes, M.; Galecki, P.; Sliwinski, T. Impact of Single Nucleotide Polymorphisms of Base Excision Repair Genes on DNA Damage and Efficiency of DNA Repair in Recurrent Depression Disorder. Mol Neurobiol 2017, 54, 4150-4159, doi:10.1007/s12035-016-9971-6.

[8] Schormann, N.; Ricciardi, R.; Chattopadhyay, D. Uracil-DNA glycosylases-Structural and functional perspectives on an essential family of DNA repair enzymes. Protein Sci. 2014, 23, 1667-1685, doi:10.1002/pro.2554.

[9] Zhang, W.; Liu, Z.; Crombet, L.; Amaya, M. F.; Liu, Y.; Zhang, X.; Kuang, W.; Ma, P.; Niu, L.; Qi, C. Crystal structure of the mismatchspecific thymine glycosylase domain of human methyl-CpG-binding protein MBD4. Biochem. Biophys. Res. Commun. 2011, 412, 425428, doi:10.1016/j.bbrc.2011.07.091 